\title{
The Use of Indirect Resin Composites in Clinical Practice: A Case Series
}

\section{Petropoulou $A^{1 *}$, Pantzari $F^{1}$, Nomikos $\mathbf{N}^{1}$, Chronopoulos $\mathbf{V}^{2}$ and Kourtis $\mathbf{S}^{1}$}

${ }^{1}$ Department of Prosthodontics, School of Dentistry, National and Kapodistrian University of Athens, Greece

${ }^{2}$ School of Dentistry and Oral Health, Griffith University, Gold Coast, QLD, Australia

\begin{abstract}
Since the first application of indirect composite resins, numerous advances in adhesive dentistry have been made. Furthermore, improvements in structure, composition and polymerization techniques led to the development of a second-generation of indirect resin composites (IRCs). IRCs have optimal esthetic performance, enhanced mechanical properties and reparability. Due to these characteristics they can be used for a wide range of clinical applications. IRCs can be used for inlays, onlays, crowns' veneering material, fixed dentures prostheses and removable prostheses (teeth and soft tissue substitution), both on teeth and implants. The purpose of this article is to review the properties of these materials and describe a case series of patients treated with different type of restorations in various indications.
\end{abstract}

\section{Keywords: Indirect resin composite materials}

\section{Introduction}

Composite resins are used daily in clinical practice. Initially the resin composites were applied in direct restorations, but since the ' $80 \mathrm{~s}$ indirect resin composites (IRCs) were also introduced in Dentistry [1]. Later, in 1990, in order to enhance their mechanical and optical properties a second generation indirect composite resins was developed with differences in structure, composition, polymerization technique and filler reinforcement. These materials have microhybrid fillers with a diameter of 0.04-1 $\mu \mathrm{m}$ [2]. Their filler content is much higher than in the first generation of IRCs, in order to improve elasticity and strength and to reduce the organic resin matrix, resulting in reduced polymerization shrinkage [1]. Various polymerization techniques combining light, heat, vacuum or pressure are utilized to allow a higher degree of conversion of IRCs [2-5]. All the above mentioned contributed to enhanced properties of the second generation of indirect composite resins.

Several studies [5-9] were initiated to assess the clinical performance of IRCs. Compared to ceramic materials, IRCs exhibit better stress distribution [10], repairability, lower cost and ease of handling [11]. On the other hand, they show inferior long term surface characteristics, such as surface roughness and esthetics and they are more prone to color changes [2,3]. Moreover, when compared to direct composites, their advantages are esthetics, color stability and reduced postoperative sensitivity $[7,8]$. The adhesive cementation of restorations fabricated from IRCs, by means of dual curing cements, minimizes the marginal gap and compensates for the unavoidable polymerization shrinkage. Additionally, it is easier to achieve ideal proximal contacts and anatomic morphology using indirect restorations [11].

Based on their characteristics, IRCs cover nowadays a wide range of indications, including inlays, onlays, overlays, short-span fixed denture prostheses (FDP), veneering material of FDS's and removable dentures and as a repair material for a variety of restorations.

The aim of this paper is to review some of the applications of these materials and present clinical cases to illustrate the scope of utilizations of IRCs.

\section{Case A}

A 45-year-old Caucasian male patient was referred to the Postgraduate Clinic of Prosthodontics (Dental School, National and Kapodistrian University of Athens). The maxillary left first molar had an amalgam filling with secondary caries at the palatal margins (Figure 1). After removal of the amalgam and caries, the enamel of the palatal cusp was evaluated as unsupported and had to be removed (Figure 2). The pulp was vital but the remaining tooth structure was considered inadequate for a new direct resin composite or amalgam filling. The question was, whether the vitality of the tooth should be sacrificed to place a post to retain a core and a crown. In the concept of minimally invasive restoration, the treatment of choice was an

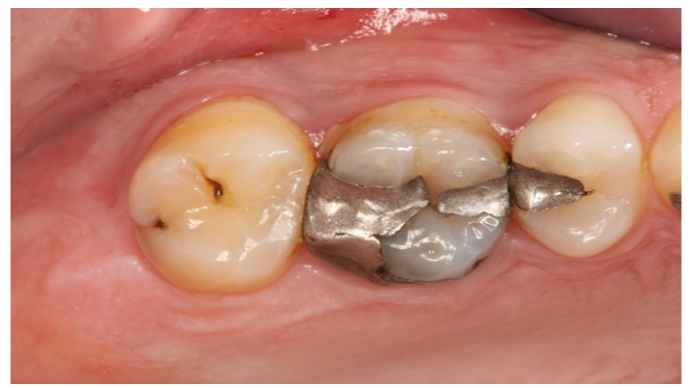

Figure 1: Case A. Initial clinical situation of first maxilarry left molar.

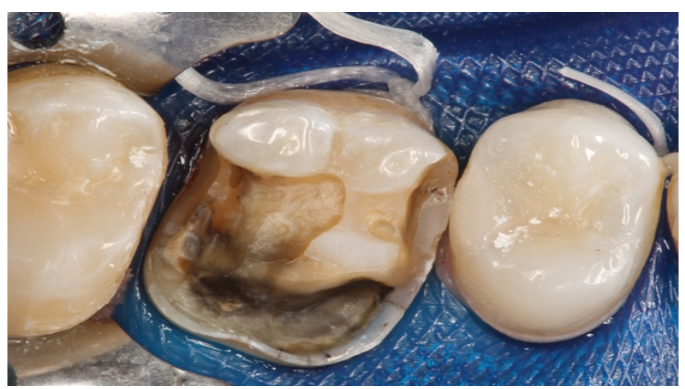

Figure 2: Preparation for onlay.

*Corresponding author: Aikaterini Petropoulou, Clinical Instructor, Department of Prosthodontics, School of Dentistry, National and Kapodistrian University of Athens, Greece, Tel: +306932989104; E-mail: aikatpetropoulou@gmail.com

Received November 10, 2013; Accepted November 28, 2013; Published November 30, 2013

Citation: Petropoulou A, Pantzari F, Nomikos N, Chronopoulos V, Kourtis S (2013) The Use of Indirect Resin Composites in Clinical Practice: A Case Series. Dentistry 3: 173. doi:10.4172/2161-1122.1000173

Copyright: ( 2013 Petropoulou A, et al. This is an open-access article distributed under the terms of the Creative Commons Attribution License, which permits unrestricted use, distribution, and reproduction in any medium, provided the original author and source are credited. 
indirect resin composite onlay (Solidex, Shofu; Kyoto, Japan) (Figure 3 ). Consequently, the root canal therapy could be avoided and the maximum amount of dental tissue could be preserved. The onlay was cemented on the tooth with dual polymerization resin cement (Panavia F 2.0, Kuraray; Tokyo, Japan) following the manufacturer's instructions (Figure 4).

\section{Case B}

In restoring patients using dental implants, the selection of the interim restoration is of a great importance, especially in the esthetic zone, since it can ensure adequate function and esthetics for the patient and can be used as a guide for the final restoration. For the replacement of anterior teeth, tooth-supported fixed denture prosthesis (Rochette Type Bridge) can be a favorable choice to eliminate pressure on the surgical site [12-14].

A 20-year-old Caucasian male patient was referred for restoration to the Post-graduate Clinic of Prosthodontics (Dental School, National and Kapodistrian University of Athens), after a severe accident, which led to traumatic luxation of the four maxillary incisors (Figure 5 and 6). A long-term provisional restoration was fabricated for immediate esthetic and functional rehabilitation. The selected restoration was a resin-bonded partial-coverage FDP retained by the intact maxillary canines and first premolars (Rochette Type Bridge) (Figure 7) $[15,16]$.

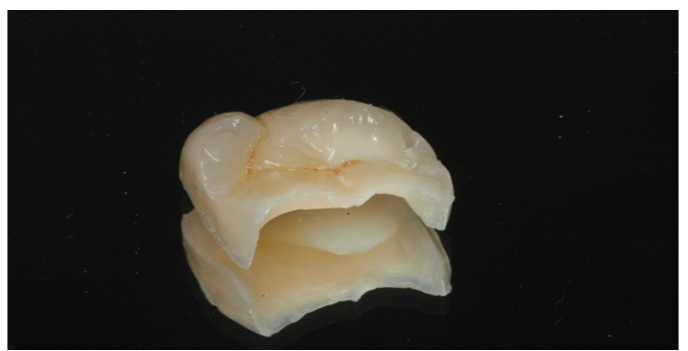

Figure 3: IRC onlay.

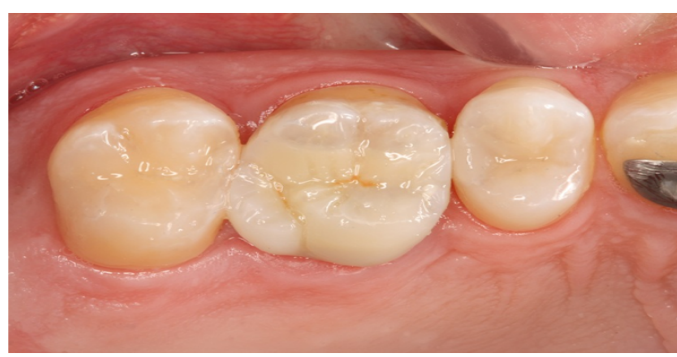

Figure 4: Onlay after cementation

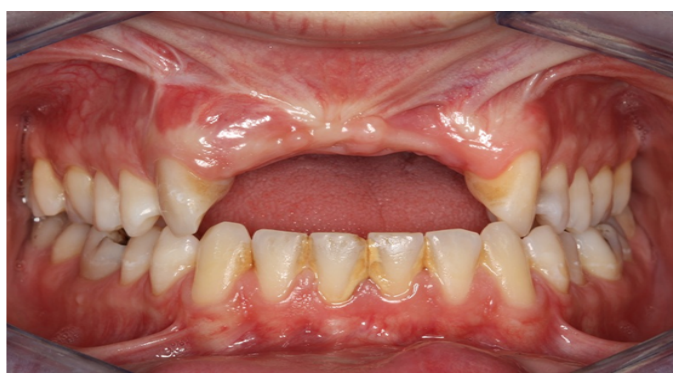

Figure 5: Case B. Initial clinical situation. Buccal view.

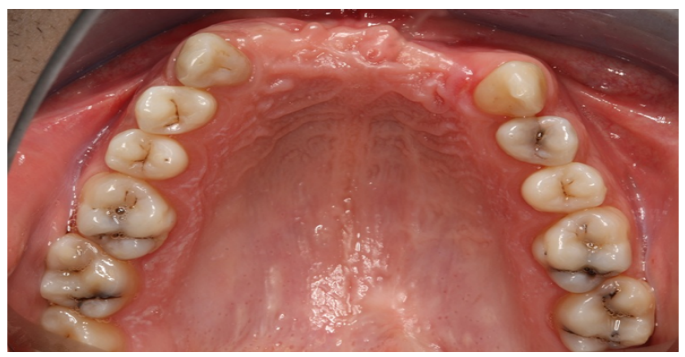

Figure 6: Initial clinical situation. Occlusal view,

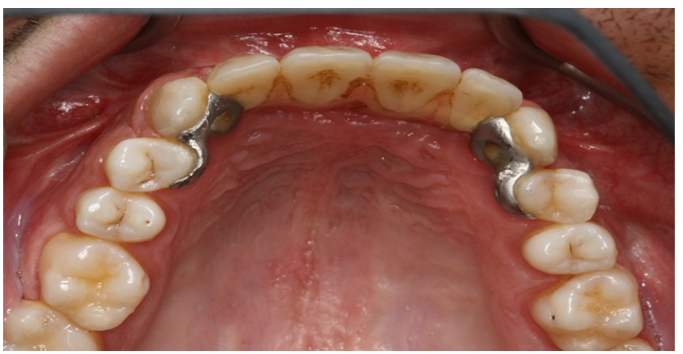

Figure 7: Long term provisional. Buccal view,

Preparation of the teeth on the palatal side was avoided due to existence of adequate inter-arch space. The Rochette Bridge restored the missing teeth and the soft tissues as well. The FDP had a metal framework and acrylic denture teeth which were veneered with tooth-colored IRC (Gradia, GC Europe N.V.; Leuven, Belgium) and gingiva-colored composite was used (Gradia Gum, GC Europe N.V.; Leuven, Belgium) for soft tissues imitation (Figure 8). The esthetic outcome fully satisfied the young patient.

\section{Case C}

Even though implants have become a more common treatment in everyday clinical practice and most patients demand fixed restorations, there are still cases where a removable prosthesis may be the treatment of choice [17]. Despite the evolution of materials and techniques, the majority of removable restorations still look unnatural. Therefore, the esthetics of removable prosthesis can be challenging for the clinician.

A 65-year-old Caucasian male patient was referred to the Postgraduate Clinic of Prosthodontics (Dental School, National and Kapodistrian University of Athens) (Figure 9). A removable partial denture with a combination of an attachment and clasps, and two metal-ceramic crowns at the maxilla were constructed according to the treatment plan. In this clinical case IRCs (Gradia and Gradia Gum, GC Europe N.V.; Leuven, Belgium) were used not only at the flanges but also as veneering material over the prefabricated denture teeth. An adequate amount of acrylic material was removed both from the labial surface of the acrylic flanges and the teeth in order to obtain enough space for the resin [18]. Thus, a natural appearance using both tooth- and gingivalcolored resin composite was achieved through customization of the removable prostheses (Figure 10-12).

\section{Case D}

A 65-year Caucasian female patient presented for the one-year recall to the Post-graduate Clinic of Prosthodontics (Dental School, National and Kapodistrian University of Athens). The patient had a 


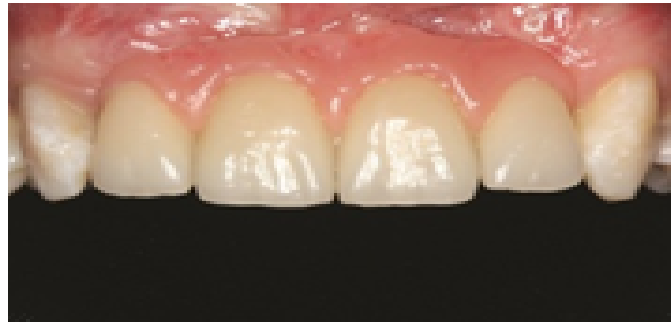

Figure 8: Long term provisional. Occlusal view.

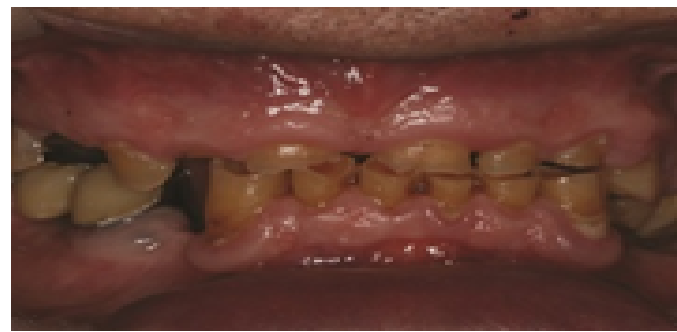

Figure 9: Case C. Initial clinical situation.

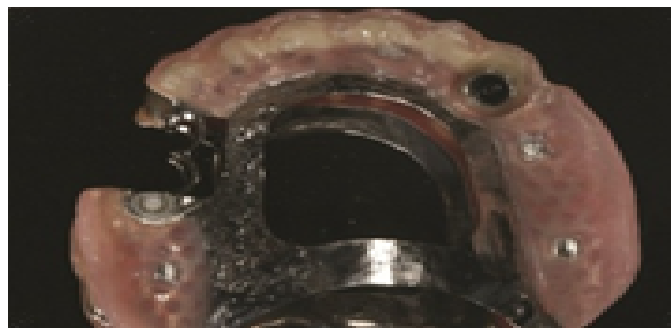

Figure 10: Removable partial denture.

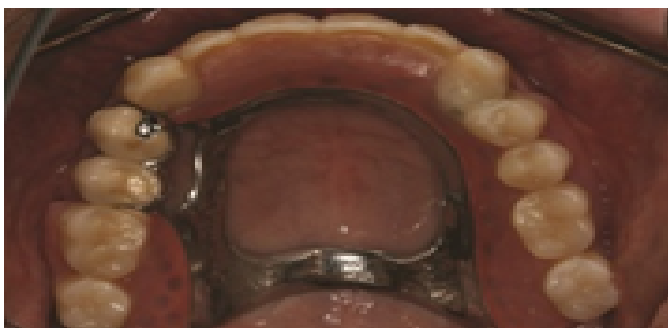

Figure 11: Removable partial denture. Intaglio surface.

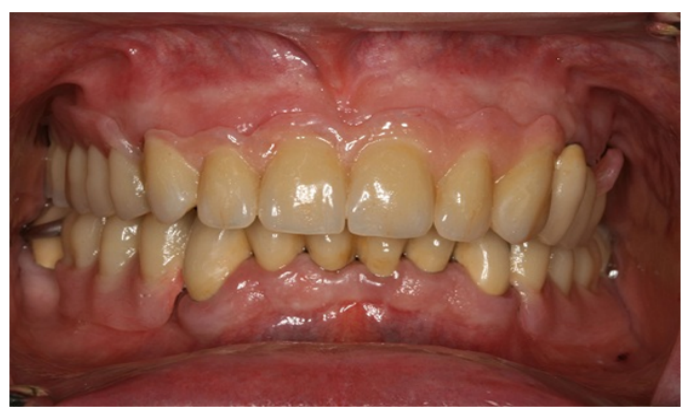

Figure 12: Final restorations. metal-ceramic FDP on implants \#16-13, a metal- ceramic FDP on teeth \#12-25 and a screw-retained implant supported full arch prostheses on the edentulous mandible extending from $\# 35$ to $\# 45$. After the initial clinical and radiographic evaluation, the patient was diagnosed with root fractures on teeth \#12,24 that were treated with gold posts. Both teeth were hopeless and had to be extracted. Fractures and cracks were also observed on the veneering porcelain of the implant supported FDP in \#16-13. The patient reported bruxing during day and night and admitted being under psychological stress and not using her occlusal guard regularly.

The treatment plan included extraction of the fractured teeth and insertion of 4 implants in the area \#12-25 (Figure 13). The new restoration was a cement-retained FDP on implants \#12,11, 14, 25, where the veneering material was IRC on a metal framework (Figures 14 and 15). The existing restorations were removed (\#16-13, \#35-45) and the ceramic veneering was replaced with IRCs (Solidex, Shofu; Kyoto, Japan) (Figure 16).

The patient's bruxing habits were controlled during the whole period of treatment with interim occlusal guards at all stages of

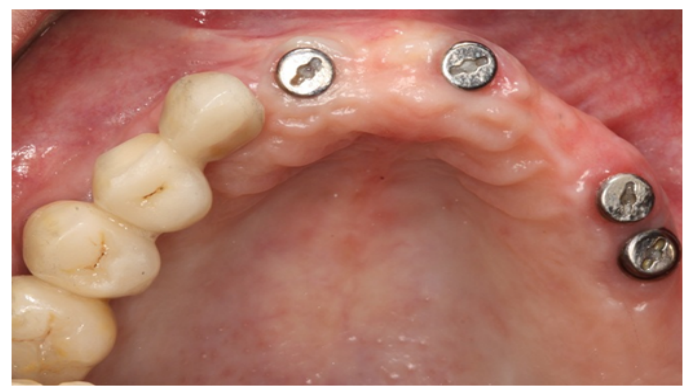

Figure 13: Case D. Initial clinical situation.

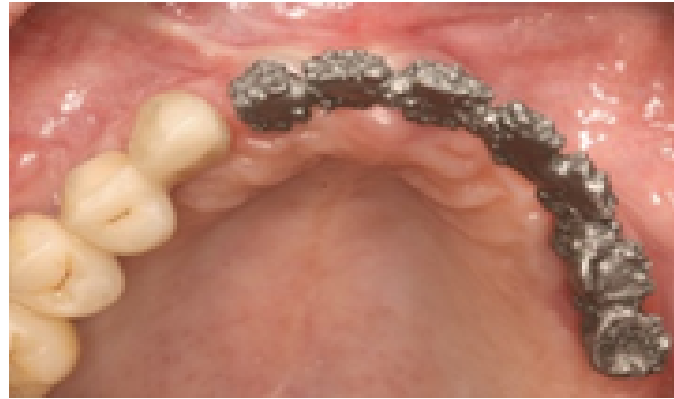

Figure 14: Metal framework on implants.

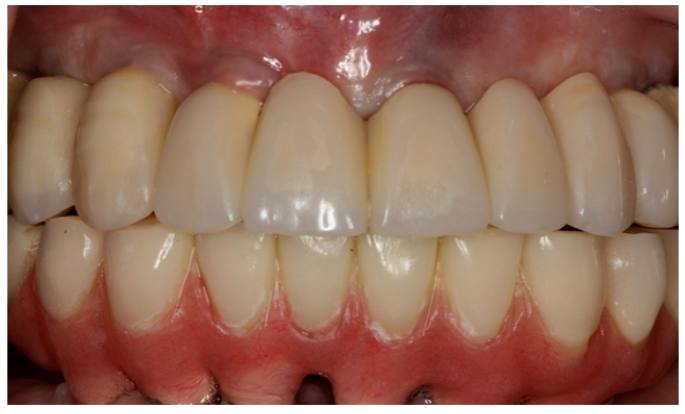

Figure 15: Final prosthesis. Occlusal view. 
treatment. The patient received psychological consultation and was placed in a 4-month recall schedule. The new restorations showed no sign of wear or fracture during 2 years of clinical use.

\section{Case E}

A 58-year old Caucasian male patient presented to the Post-graduate Clinic of Prosthodontics (Dental School, National and Kapodistrian University of Athens) complaining of the retention of the mandibular complete denture. The patient had a metal-acrylic fixed restoration on the maxilla, with metal occlusal surfaces following the shortened dental arch concept (teeth\#15-25) and was a heavy bruxer.

The patient was treated in a staged-approach treatment starting with the mandible. Rehabilitation of the maxilla would be initiated after the completion of treatment of the mandible, in a second stage. Six implants (3i Osseotite external connection, Biomet 3i; Warsaw, Indiana) were placed in the intraforamenal area (Figure 17) and screw-retained implant supported prosthesis was manufactured. Multi-unit abutments were screwed on the implants and a cast base metal alloy framework was

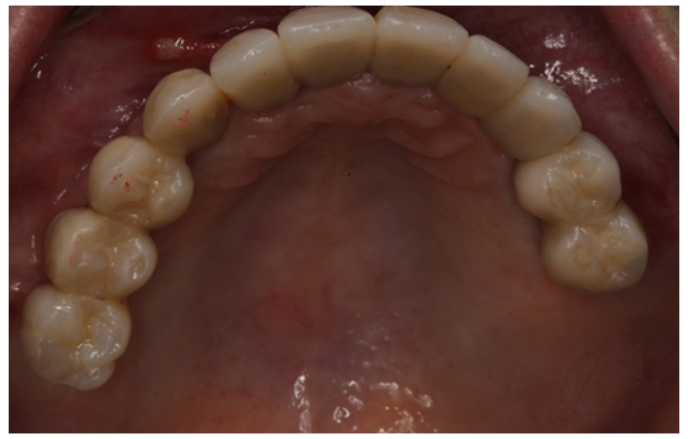

Figure 16: Final prosthesis. Buccal view.

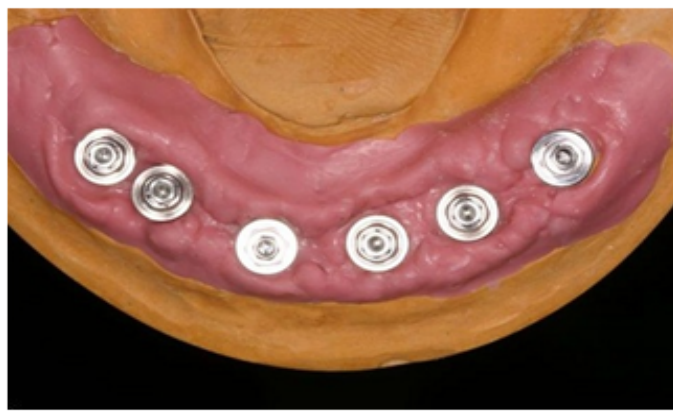

Figure 17: Case E. Working cast with implant analogs.

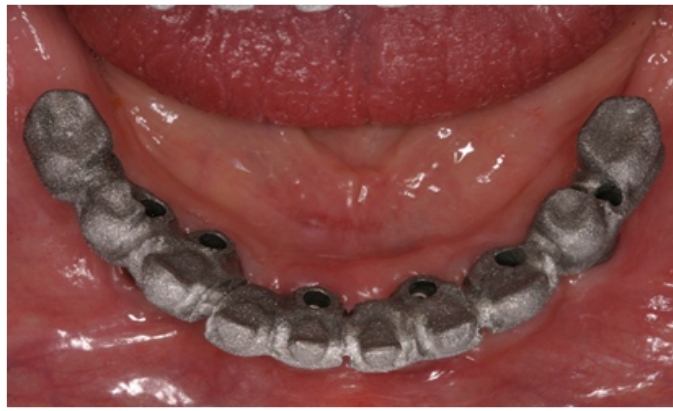

Figure 18: Metal framework try-in.

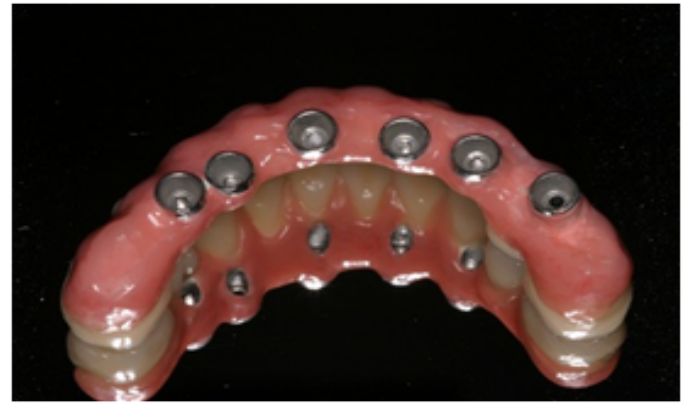

Figure 19: Screw-retained metal-polymer FPD. Intaglio surface.

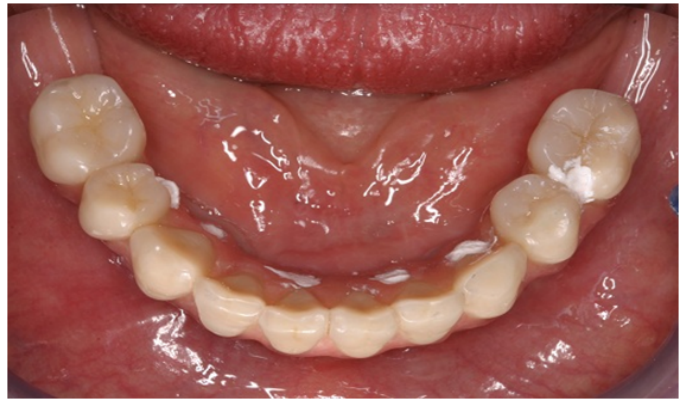

Figure 20: Final prosthesis.

fabricated on them (Figure 18). The passive fit was tested with Sheffield test on the implant \#35. The prosthesis was veneered with tooth-colored IRC for teeth (Gradia, GC Europe N.V.; Leuven, Belgium) and gingivacolored IRC for the soft tissues (Gradia Gum, GC Europe N.V.; Leuven, Belgium) (Figures 19 and 20).

Choosing IRCs for this screw-retained prosthesis offers the clinician the possibility to retrieve this prosthesis if considered necessary during the treatment of the maxilla and add or remove material in order to achieve optimal occlusal contacts or to repair possible fractures of the material.

\section{Discussion}

In several clinical cases, the IRCs can be used to modify existing prostheses or to replace metal-ceramic restorations. Fractures on the existing metal-ceramic restorations are a common finding in bruxing patients. The lack of adequate repairability intraorally and the need of replacement of the metal-ceramic restorations is time consuming and remains a financial burden for the patient. In these cases the IRCs offer the patient better stress distribution $[19,20]$, functional and esthetical results similar to ceramics, as in the presented Case $\mathrm{C}$ and the option to repair future fractures intraorally.

The ultra fine compact filled composite resin that is used in these prostheses, demonstrates wear not statistically different from that of human enamel [20]. Focusing on stress distribution, the IRCs seem to reduce the impact force under static and dynamic load when compared to metal-ceramic restorations [19].

Another advantage of IRCs is the variety of color combinations for teeth and gingiva, which offers the clinician the opportunity to obtain an optimal white and pink esthetic outcome. In clinical cases B, C and E the use of both tooth- and gingiva-colored IRCs led to natural looking restorations. 
According to an in vitro study conducted by Magne et al. [21], indirect onlays seem to be the treatment of choice for extended restorations, including cusp coverage, in vital teeth. IRCs exhibit good mechanical properties, greater capacity to distribute tensions in a more homogeneous way than ceramics [10], and reduced risk during try-in [11]. Additionally, they have lower cost than ceramic materials and several researches have reported that they present a good success and survival rate $[22,23]$ qualifying them as a competitive restorative procedure in stress-bearing regions as in Case A.

A matter of concern is the bond between IRCs and the underlying metal alloy or ceramic material. The bonding between composite resins and metal alloys is based mainly on micromechanical retention [24]. New metal- resin bonding techniques promise a stronger chemical bond between resin and base $[25,26]$ or Ti-alloys $[27,28]$. Furthermore, composite resins with chemically active monomers that bond directly to the surface of airborne-particle abraded alloys by means of various bonding agents lead to some kind of chemical bond [24]. The creation of a proper metal-resin interface can significantly improve the resinalloy shear bond strength [24,29]. As far as zirconia is concerned, Komine et al. [30] concluded that airborne-particle abrasion leads to increased bond strength between IRCs and zirconia ceramics. It is still unclear whether the use of micromechanical retention is mandatory or not. According to some manufacturers' instructions airborne-particle abrasion and application of a specific metal alloy primer may provide a durable bond between metal framework and IRCs (GC, SR Adoro).

A common disadvantage referred in the literature is their unpredictable color stability and translucency [31,32]. However, in several in-vitro trials the measured color changes were within the clinically acceptable range [31-34].

As mentioned before, the use of indirect composite resins for a wide range of applications has been increasing, mainly as a result of their improved mechanical properties, convenient handling, favorable esthetics and abrasion similar to natural hard tissues. IRCs are used with increasing frequency as a viable alternative to porcelain and direct composite, with promising clinical results [22,23,35-37].

In some in-vitro studies, it has been reported that IRCs designed for the same applications presented different mechanical behavior, especially as far as wear and hardness were concerned [5,38]. Mandikos et al. [38] propose that despite their wear and hardness difference, IRCs can perform satisfactorily under clinically induced conditions of wear.

However, there are not enough clinical trials on these materials for all clinical applications. Dukic et al. [22] evaluated 71 indirect resin composite restorations (onlays) on permanent premolars after 36 months in use. They concluded that IRCs restorations can be a good treatment option for severely damaged teeth. Huth et al. [23] evaluated IRCs inlays placed by students, after 4 years. The researchers used the same criteria as the previous ones, the modified USPHS criteria. They found that after 4 years, the clinical acceptable restorations vary from $76.6 \%$ to $87.2 \%$ depending on the different IRCs brand. Hence, IRCs restorations seem to be a viable treatment option in stress-bearing preparations. Jongsma et al. [35] evaluated forty-five patients with 86 IRCs crowns and 5 onlays after 3 years of clinical performance. They concluded that IRCs restorations exhibit $91.6 \%$ survival rate and $84.8 \%$ success rate.

Consequently, more in-vitro and especially clinical trials are needed to verify the longevity of IRCs in different clinical indications. On the opposite side IRCs seem to be an alternative choice as restorative or veneering material in selected clinical cases.

\section{Disclosure}

The authors do not have any financial interest in the companies whose products are included in this article.

\section{References}

1. Miara P (1998) Aesthetic guidelines for second-generation inlays and onlay composite restorations. Prac Periodont Aesthet Dent 10: 423-431.

2. Nandini S (2010) Indirect resin composites. J Conserv Dent 13: 184-194.

3. Hirata M, Koizumi H, Tanoue N, Ogino T, Murakami M, et al. (2011) Influence of laboratory light sources on the wear characteristics of indirect composites. Dent Mater J 30: 127-135.

4. Nishimaki M (2012) Depth of cure and hardness of indirect composite materials polymerized with two metal halide laboratory curing units. J Oral Sci 54: 121125

5. Souza RO, Ozcan M, Michida SM, de Melo RM, Pavanelli CA, et al. (2010) Conversion degree of indirect resin composites and effect of thermocycling on their physical properties. J Prosthodont 19: 218-225.

6. Cetin AR, Unlu N (2009) One-year clinical evaluation of direct nanofilled and indirect composite restorations in posterior teeth. Dent Mater J 28: 620-626.

7. Hickel R, Manhart $J(2001)$ Longevity of restorations in posterior teeth and reasons for failure. J Adhes Dent 3: 45-64

8. Touati B (1996) The evolution of aesthetic restorative materials for inlays and onlays: a review. Pract Periodontics Aesthet Dent 8: 657-666.

9. Touati B, Aidan N (1997) Second generation laboratory composite resins for indirect restorations. J Esthet Dent 9: 108-118

10. Soares CJ, Martins LR, Pfeifer JM, Giannini M (2004) Fracture resistance of teeth restored with indirect-composite and ceramic inlay systems. Quintessence Int 35: 281-286.

11. Soares CJ, Santana FR, Fonseca RB, Martins LR, Neto FH (2007) In vitro analysis of the radiodensity of indirect composites and ceramic inlay systems and its influence on the detection of cement overhangs. Clin Oral Investig 11 : 331-336.

12. Conte GF, Fagan MC, Kao RT (2008) Provisional restorations: a key determinant for implant site development. J Calif Dent Assoc 36: 261-267.

13. Priest G (2006) Esthetic potential of single-implant provisional restorations: selection criteria of available alternatives. J Esthet Restor Dent 18: 326-338.

14. Poggio CE, Salvato A (2002) Bonded provisional restoration for esthetic soft tissue support in single-implant treatment. J Prosthet Dent 87: 688-691.

15. Banerji S, Sethi A, Dunne SM, Millar BJ (2005) Clinical performance of Rochette bridges used as immediate provisional restorations for single unit implants in general practice. Br Dent J 199: 771-775.

16. Barber MW, Preston AJ (2008) An update on resin-bonded bridges. Eur $J$ Prosthodont Restor Dent 16: 2-9.

17. Zitzmann NU, Marinello CP (1999) Treatment plan for restoring the edentulous maxilla with implant supported restoration: removable overdenture versus fixed partial denture design. J Prosthet Dent 82: 188-196.

18. Patras M, Sykaras N (2012) Esthetic and functional combination of fixed and removable prostheses. Gen Dent 60: e47-54.

19. Juodzbalys G, Kubilius R, Eidukynas V, Raustia AM (2005) Stress distribution in bone: single-unit implant prostheses veneered with porcelain or a new composite material. Implant Dent 14: 166-175

20. Mehl C, Scheibner S, Ludwig K, Kern M (2007) Wear of composite resin veneering materials and enamel in a chewing simulator. Dent Mater 23: 1382 1389.

21. Magne P, Belser UC (2003) Porcelain versus composite inlays/onlays: effects of mechanical loads on stress distribution, adhesion, and crown flexure. Int $J$ Periodontics Restorative Dent 23: 543-555.

22. Dukic W, Dukic OL, Milardovic S, Delija B (2010) Clinical evaluation of indirect composite restorations at baseline and 36 months after placement. Oper Dent 35: 156-164.

23. Huth KC, Chen HY, Mehl A, Hickel R, Manhart J (2011) Clinical study of indirect composite resin inlays in posterior stress-bearing cavities placed by dental students: results after 4 years. J Dent 39: 478-488. 
Citation: Petropoulou A, Pantzari F, Nomikos N, Chronopoulos V, Kourtis S (2013) The Use of Indirect Resin Composites in Clinical Practice: A Case Series. Dentistry 3: 173. doi:10.4172/2161-1122.1000173

24. Almilhatti HJ, Giampaolo ET, Vergani CE, Machado AL, Pavarina AC, et al. (2009) Adhesive bonding of resin composite to various $\mathrm{Ni}-\mathrm{Cr}$ alloy surfaces using different metal conditioners and a surface modification system. J Prosthodont 18: 663-669.

25. Sarafianou A, Seimenis I, Papadopoulos T (2008) Effectiveness of different adhesive primers on the bond strength between an indirect composite resin and a base metal alloy. J Prosthet 99: 377-387.

26. Seimenis I, Sarafianou A, Papadopoulou H, Papadopoulos T (2006) Shear bond strength of three veneering resins to a $\mathrm{Ni}-\mathrm{Cr}$ alloy using two bonding procedures. J Oral Rehabil 33: 600-608.

27. Fernandes CA, Ribeiro JC, Larson BS, Bonfante EA, Silva NR, et al. (2009) Microtensile bond strength of resin-based composites to Ti-6Al-4V. Dent Mater 25: $655-661$.

28. Lee SY, Vang MS, Yang HS, Park SW, Park HO, et al. (2009) Shear bond strength of composite resin to titanium according to various surface treatments. J Adv Prosthodont 1: 68-74.

29. Shimoe S, Tanoue N, Satoda T, Murayama T, Nikawa H, et al. (2010) Evaluation of single liquid primers with organic sulphur compound for bonding between indirect composite material and silver-palladium-copper-gold alloy. Dent Mater J 29: 25-29.

30. Komine F, Kobayashi K, Saito A, Fushiki R, Koizumi H, et al. (2009) Shear bond strength between an indirect composite veneering material and zirconia ceramics after thermocycling. J Oral Sci 51: 629-634.
31. Douglas RD (2000) Color stability of new-generation indirect resins for prosthodontic application. J Prosthet Dent 83: 166-170.

32. Papadopoulos T, Sarafianou A, Hatzikyriakos A (2010) Colour stability of veneering composites after accelerated aging. Eur J Dent 4: 137-142.

33. Nakamura T, Saito O, Mizuno M, Tanaka H (2002) Changes in translucency and color of particulate filler composite resins. Int J Prosthodont 15: 494-499.

34. Stawarczyk B, Egli R, Roos M, Ozcan M, Hämmerle CH (2011) The impact of in vitro aging on the mechanical and optical properties of indirect veneering composite resins. J Prosthet Dent 106: 386-398.

35. Jongsma LA, Kleverlaan CJ, Feilzer AJ (2012) Clinical success and survival of indirect resin composite crowns: results of a 3-year prospective study. Dent Mater 28: 952-960

36. Monaco C (2012) A clinical case report on indirect, posterior three-unit resinbonded FRC FPD. J Adhes Dent 14: 479-483.

37. Kharade P, Sharma S, Banerjee A, Gupta T (2012) Indirect resin-bonded fiberreinforced composite anterior bridge: A case report. Gen Dent 60: e170-172.

38. Mandikos MN, McGivney GP, Davis E, Bush PJ, Carter JM (2001) A comparison of the wear resistance and hardness of indirect composite resins. J Prosthet Dent 85: 386-395. 